\title{
Casson fluid flow with heat and mass transfer in a channel using the differential transform method
}

\author{
Asia Yasmin ${ }^{1}$, Kashif Ali ${ }^{2}$, Muhammad Ashraf ${ }^{1, *}$ \\ ${ }^{1}$ Centre for Advanced Studies in Pure and Applied Mathematics, \\ Bahauddin Zakariya University Multan, Pakistan \\ ${ }^{2}$ Dept. of Basic Sciences and Humanities, \\ Muhammad Nawaz Sharif University of Engineering and Technology, Multan. \\ *Corresponding author: muhammadashraf@bzu.edu.pk
}

\begin{abstract}
In the present investigation, we consider the heat and mass transfer characteristics of steady, incompressible and electrically conducting Casson fluid flow in a channel. The effect of chemical reactions have also been considered. The differential transform method (DTM) is applied to a system of non-linear ODEs, and the results are obtained in the form of DTM series. The principal gain of this approach is that it applies to the non-linear ODEs without requiring any discretization, linearization or perturbation. The velocity, mass and heat transfer profiles thus obtained are in good agreement with those provided by the quasi-linearization method (QLM). Graphical results for velocity, concentration and temperature fields are presented for a certain range of values of the governing parameters.
\end{abstract}

Keywords: Casson fluid; channel flow; differential transform method (DTM); shrinking wall; quasi-linearization method (QLM)

\section{Introduction}

Non-Newtonian fluid flow has been the objective of much research due to its increasing industrial and technological applications. Many fluids like drilling mud, polymer solutions, paints, shampoo, ketchup etc. do not obey the Newton's law for viscosity. Hence, the Navier-Stokes theory is not able to explain the behaviour of these fluids and there is also no single equation in literature that represents properties for this type of fluids. Casson fluid model (preferred rheological representation for chocolate and blood) was presented by Casson for the flow of viscoelastic fluids in 1959. It is the main non-Newtonian fluid that exhibits yield stress, and have many applications in metallurgy, drilling operations, bio-engineering operations and food processing.

Eldabe et al. (2014) considered the peristaltic movement of non-Newtonian fluid with mass and heat transfer with in a porous medium in a channel under an external magnetic field. Ahmed (2006) investigated the heat transfer properties of both cold side walls and hot fins and buoyancy nature of a flow inside an enclosure. The entrance region flow of a Casson fluid in a straight channel was examined numerically by Batra \& Kandasamy (2006). The mass and heat transfer characteristics of magnetohydrodynamics Casson fluid in upright stretching walls channel was investigated by Sarojamma et al. (2014) using the shooting technique. Ramesh \& Devakar (2015) obtained the interpretive solutions for flow of Casson fluid within parallel plates under slip settings. Raju et al. (2016) discussed the mass and heat transfer behaviour for Casson fluid past 
exponentially porous stretching surface in the occurrence of magnetic field, thermal emission, chemical reaction, viscous dissipation and heat source. In this study, dual solutions were presented for the Casson fluid, contrary to the Newtonian flow. The consequence of thermal radiation on mass and heat transfer of MHD flow for Casson fluid above a stretching surface was considered by Haritha \& Sarojamma (2014). Hafiz et al. (2016) studied the heat transfer and mixed convection in the Powell-Eyring flow with Newtonian heating, over a stretching cylinder. They conclude that, by increasing the Prandtl number and convection parameter, the temperature distribution decreases significantly whereas the flow may be accelerated by increasing the curvature parameter.

Channel flows have applications in the fields of filtration, grain regression, surface sublimation, ablation cooling, microfluidic devices and binary gas diffusion, see Ashraf et al. (2009). Numerous studies have been presented on channel flow since the pioneering study by Berman in 1953. Recent investigations in this field are Si et al. (2010); Ali et al. (2014); Sheikholeslami et al. (2014); Ali \& Asharf(2014) and Ali et al. (2015). Three iterative techniques (TAM, BCM and DJM) have been used by Majeed \& Al-Zahraa (2020) for the approximate solution of the Jeffery-Hamel flow problem. They obtained approximate solutions in the form of convergent series in which no restriction assumptions were applied for nonlinear terms. Moreover, the obtained results were compared with Runge-Kutta technique.

A large number of phenomena are basically non-linear and are modelled by non-linear equations. Several methods have been established to solve non-linear equations. Differential transform method (DTM) is one of the methods that do not require small parameters. This method has been effectively applied to many application problems (Hassan, 2002; Momania \& Ert"urk, 2008; Abazari \& Borhanifar, 2010; Fereidoom et al., 2010; Joneidi et al., 2009). The first use of DTM was in the solution of electrical circuit analysis by Zhou (1986). Chen \& Ho (1999) presented this technique for PDEs whereas Ayaz (2004) applied it to a system of differential equations. To find out the exact solution of linear and non-linear space time fractional reaction diffusion equations on a finite domain, Mridula \& Pratibha (2013) have used the generalized differential transform technique. Through this method, the drawbacks of homotopy perturbation method and the domain decomposition techniques have been overcome. For further details on DTM, we highly recommend the work by Mridula \& Pratibha (2013).

In this paper, we study the flow of Casson fluid accompanied by heat and mass transfer through channel with a stationary and a shrinking wall with the slip condition, in the presence of chemical reaction. A similarity transformation is utilized to transfer governing equations into a set of non-linear ODEs which are then solved by employing the differential transform method (DTM) and quasi-linearization method (QLM). Impact of the governing parameters on flow, heat and mass transfer features of the problem are explained.

\section{Mathematical Formulation}

We take into account the magnetohydrodynamic (MHD) flow of Casson fluid in a parallel plate channel with a shrinking lower wall and a stationary upper wall, in the presence of applied magnetic field. It is assumed that the induced magnetic field is in significant in comparison with the imposed one. The magnetic Reynolds number $(R e)$ is taken small. For small $R e$, magnetic field will have a tendency to settle down towards simply diffusive state. Also, it is assumed that there 
is no electric polarization, and subsequently no electric field. The channel walls are positioned at $y= \pm a$.Consequently, we may express the velocity $\underline{v}$ as $\underline{v}=(u(x, y), v(x, y), 0)$

Figure 1 shows the physical model of the problem.


Fig. 1. Physical model for the problem.

By following Sarojamma et al. (2014), governing equations for flow, heat and mass transfer in viscous and incompressible flow are:

$$
\begin{aligned}
& \frac{\partial u}{\partial x}+\frac{\partial v}{\partial y}=0, \\
& u \frac{\partial u}{\partial x}+v \frac{\partial u}{\partial y}=\frac{-1}{\rho} \frac{\partial p}{\partial x}+\frac{\mu}{\rho}\left(1+\frac{1}{\beta}\right) \nabla^{2} u-\frac{\sigma B_{0}^{2}}{\rho} u, \\
& u \frac{\partial v}{\partial x}+v \frac{\partial v}{\partial y}=\frac{-1}{\rho} \frac{\partial p}{\partial y}+\frac{\mu}{\rho}\left(1+\frac{1}{\beta}\right) \nabla^{2} v, \\
& \rho c_{p}\left(u \frac{\partial T}{\partial x}+v \frac{\partial T}{\partial y}\right)=k_{0} \frac{\partial^{2} T}{\partial y^{2}}, \\
& u \frac{\partial C}{\partial x}+v \frac{\partial C}{\partial y}=D \frac{\partial^{2} C}{\partial y^{2}}-K_{1}\left(C-C_{2}\right) .
\end{aligned}
$$

Here, $p$ is the pressure, $\rho$ is the density, $\mu$ is the dynamic viscosity, $\beta$ is the Casson fluid parameter, $\sigma$ is electrical conductivity, $B_{0}$ represents the magnetic field strength, $c_{p}$ is the specific heat at constant pressure, $T$ is the temperature, $k_{0}$ is the thermal conductivity, and $C$ is the concentration. Further, $K_{1}$ is the first order chemical reaction rate, and $D$ is the molecular diffusivity. The boundary conditions are as follows: 


$$
\left.\begin{array}{l}
u(x,-a)=-b x+A \frac{\partial u}{\partial y}, u(x, a)=0, v(x, \pm a)=0, T(x,-a)=T_{1}, \\
T(x, a)=T_{2}, C(x,-a)=C_{1}, C(x, a)=C_{2}
\end{array}\right\}
$$

where $T_{1}, T_{2}$ and $C_{1}, C_{2}$ are the fixed temperatures and concentrations at the lower and upper walls, respectively. Further, $b>0$ is the shrinking rate of the lower channel wall, and $A$ is the velocity slip factor. It is important to point out that such slip conditions have been considered by many researchers (Wang, 2009; Fang et al., 2009; Mukhopadyay \& Andersson, 2009; Bhattacharyya et al., 2011; Aman et al., 2011; Fang et al., 2010). Slip flow is vital in microelectromechanical structures (MEMS) where the flow pattern is noticeably different from the conventional no slip flow due to the microscale dimensions of these devices.

We introduce subsequent similarity variables:

$$
\eta=\frac{y}{a}, u=b x f^{\prime}(\eta), v=-a b f(\eta), \theta(\eta)=\frac{T-T_{2}}{T_{1}-T_{2}}, \phi(\eta)=\frac{C-C_{2}}{C_{1}-C_{2}} .
$$

It is to point out that equation (8) represents a similarity transformation in which the velocity, temperature and concentration fields are defined in terms of a new dimensionless variable $\eta$ (which depends on $y$ only) in such a way that the continuity equation is identically satisfied, and the mathematical model reduces to a set of non-linear ODEs. Further, $f$ and $f^{\prime}$ ( derivative of $f$ w.r.t $\eta$ ) represent the dimensionless streamwise and normal velocity fields. It is to point out that the use of similarity transformation is not rare, and there are numerous studies (for example, Ashhab, 2019; Wahab et al., 2016) where such a powerful tool has been employed.

After eliminating pressure term from equations (3) - (4), and using (8), we get,

$$
\left(1+\frac{1}{\beta}\right) f^{i v}+\operatorname{Re}\left(f f^{\prime \prime \prime}-f^{\prime} f^{\prime \prime}\right)-M f^{\prime \prime}=0
$$

Whereas, equations (5) and (6) acquire the forms:

$$
\begin{aligned}
& \theta^{\prime \prime}+\operatorname{Pr} \operatorname{Ref} \theta^{\prime}=0, \\
& \phi^{\prime \prime}-\operatorname{Re} \operatorname{Sc}\left(\operatorname{Cr} \phi-f \phi^{\prime}\right)=0 .
\end{aligned}
$$

Now, $\operatorname{Re}=\frac{\rho a^{2} b}{\mu}, M=\frac{a^{2} \sigma B_{0}{ }^{2}}{\mu}, \operatorname{Pr}=\frac{\mu c_{p}}{\kappa_{0}}, C r=\frac{k_{1}}{b}$ and $S c=\frac{v}{D}$ represent the Reynolds number, the magnetic parameter, the Prandtl number, the chemical reaction parameter and the Schmidt number, respectively. The related boundary conditions (7) become:

$$
f( \pm 1)=0, f^{\prime}(1)=0, f^{\prime}(-1)=-1+\lambda f^{\prime \prime}, \theta(-1)=1, \theta(1)=0, \phi(-1)=1, \phi(1)=0,
$$

where $\lambda=\frac{A}{a}$ is the velocity slip parameter. 


\section{Solution Methodology}

System of ODEs (9-11) is solved by employing two different techniques, one is the differential transformation method (DTM), and the other is the quasi-linearization method (QLM). DTM is based on the Taylor series expansion and provides the solution in the form of polynomial series whereas the QLM gives numerical solution.

\subsection{Differential Transform Method (DTM)}

DT (differential transform) of function $h(\eta)$ is described as follows:

$$
H(s)=\frac{1}{s !}\left[\frac{d^{s} h(\eta)}{d \eta^{s}}\right]_{\eta=\eta_{0}},
$$

where $H(s)$ is transformed function for the original function $h(\eta)$. Inverse DT of $H(s)$ is defined as

$$
h(\eta)=\sum_{s=0}^{\infty} H(s)\left(\eta-\eta_{0}\right)^{s} .
$$

Combining equations (13) and (14), we get

$$
h(\eta)=\sum_{s=0}^{\infty}\left[\frac{d^{s} h(\eta)}{d \eta^{s}}\right]_{\eta=\eta_{0}} \frac{\left(\eta-\eta_{0}\right)^{s}}{s !} .
$$

equation (15) demonstrates the fundamental idea of DTM that is consequent from the Taylor series expansion.

\subsection{Implementation of the DTM}

For the coupled non-linear differential equations (9-11), we first write:

$$
f(\eta)=\sum_{l=0}^{n} F(l) \eta^{l}, \theta(\eta)=\sum_{l=0}^{n} \tilde{\theta}(l) \eta^{l} \text { and } \phi(\eta)=\sum_{l=0}^{n} \tilde{\phi}(l) \eta^{l}
$$

where $F(l), \tilde{\phi}(l)$ and $\tilde{\theta}(l)$ are the transformed functions for $f(\eta), \phi(\eta)$ and $\theta(\eta)$ respectively. In view of basic properties of the DTM, we have the subsequent system:

$$
\begin{aligned}
& \left(1+\frac{1}{\beta}\right)(l+4)(l+3)(l+2)(l+1) F(l+4)+\operatorname{Re} \sum_{m=0}^{l}\left\{\begin{array}{l}
(l+3-m)(l+2-m)(l+1-m) \\
F(l-m+3) F(m)- \\
(m+1)(l+2-m)(l+1-m) \\
F(m+1) F(l-m+2)
\end{array}\right\} \\
& -M(l+2)(l+1) F(l+2)=0, \\
& (l+2)(l+1) \tilde{\theta}(l+2)+\operatorname{RePr} \sum_{m=0}^{l}(m+1) \tilde{\theta}(m+1) F(l-m)=0, \\
& (l+1)(l+2) \tilde{\phi}(l+2)-\operatorname{Re} \operatorname{Sc} \operatorname{Cr} \tilde{\phi}(l)+\operatorname{ReSc}\left(\sum_{m=0}^{l} F(m)(l-m+1) \tilde{\phi}(l-m+1)\right)=0,
\end{aligned}
$$


whereas the boundary conditions (12) are transformed into:

$$
\left.\begin{array}{l}
\sum_{l=0}^{n} l F(l)(-1)^{(l-1)}=-1+\lambda \sum_{l=0}^{n} l(l-1) F(l), \sum_{l=0}^{n} F(l)(-1)^{l}=0, \quad \sum_{l=0}^{n} \tilde{\theta}(l)(-1)^{l}=1, \\
\sum_{l=0}^{n} \tilde{\phi}(l)(-1)^{l}=1, \sum_{l=0}^{n} l F(l)=0, \sum_{l=0}^{n} F(l)=0, \sum_{l=0}^{n} \tilde{\theta}(l)=0, \sum_{l=0}^{n} \tilde{\phi}(l)=0 .
\end{array}\right\}
$$

Now let:

$$
F(0)=a_{0}, F(1)=a_{1}, F(2)=a_{2}, F(3)=a_{3}, \tilde{\theta}(0)=b_{0}, \tilde{\theta}(1)=b_{1}, \tilde{\phi}(0)=d_{0}, \tilde{\phi}(1)=d_{1} \text {. }
$$

The constants $\mathrm{a}_{0}$ to $\mathrm{a}_{3}, \mathrm{~b}_{0}, \mathrm{~b}_{1}, \mathrm{~d}_{0}, \mathrm{~d}_{1}$ are obtained using the transformed boundary conditions given by equation (20). The phase diagram for the DTM is given in the Figure 2, which describes how the missing constants needed for the DTM are determined iteratively. It is important to point out that $t$ (less than unity) is the under-relaxation factor needed to achieve convergence of the iterative process. Using the definition (14), for a certain set of parameters, we obtain solutions of the equations (9-11) as follows:

$$
\begin{aligned}
& f(\eta)=\sum_{l=0}^{n} F(l) \eta^{l}=-0.1267-0.1111 \eta+0.1227 \eta^{2}-0.0082 \eta^{3}+0.0034 \eta^{4}-0.0011 \eta^{5}+\ldots \\
& \theta(\eta)=\sum_{l=0}^{n} \tilde{\theta}(l) \eta^{l}=-0.0830+0.1501 \eta-0.2335 \eta^{2}+0.2831 \eta^{3}-0.2727 \eta^{4}+0.1925 \eta^{5}+\ldots \\
& \left.\phi(\eta)=\sum_{l=0}^{n} \tilde{\phi}(l) \eta^{l}=-0.4261+0.1617 \eta-0.0765 \eta^{2}+0.0128 \eta^{3}+0.0011 \eta^{4}-0.0028 \eta^{5}+\ldots\right\}
\end{aligned}
$$

\subsection{Solution by Quasi-linearization Method (QLM)}

Quasi-linearization is utilized to build sequences $\left\{f^{(k)}\right\},\left\{\theta^{(k)}\right\}$ and $\left\{\phi^{(k)}\right\}$ which converge to solutions of equations (9-11). To construct $\left\{f^{(k)}\right\}$, we first set:

$$
\varphi\left(f, f^{\prime}, f^{\prime \prime}, f^{\prime \prime \prime}, f^{i v}\right) \equiv\left(1+\frac{1}{\beta}\right) f^{i v}+\operatorname{Re}\left(f f^{\prime \prime \prime}-f^{\prime} f^{\prime \prime}\right)-M f^{\prime \prime} .
$$

Now, we linearized it about the $k^{\text {th }}$ iteration, by retaining only the first order terms, and arrive at:

$$
\begin{aligned}
& \varphi\left(f^{(k)}, f^{\prime(k)}, f^{\prime \prime(k)}, f^{\prime \prime \prime(k)}, f^{i v(k)}\right)+\left(f^{(k+1)}-f^{(k)}\right) \frac{\partial \varphi}{\partial f^{(k)}}+\left(f^{\prime(k+1)}-f^{\prime(k)}\right) \frac{\partial \varphi}{\partial f^{\prime(k)}}+\left(f^{\prime \prime(k+1)}-f^{\prime \prime(k)}\right) \frac{\partial \varphi}{\partial f^{\prime \prime(k)}} \\
& +\left(f^{\prime \prime(k+1)}-f^{\prime \prime(k)}\right) \frac{\partial \varphi}{\partial f^{\prime \prime(k)}}+\left(f^{i(k+1)}-f^{i v(k)}\right) \frac{\partial \varphi}{\partial f^{i v(k)}}=0,
\end{aligned}
$$

which simplifies to:

$$
\begin{aligned}
& \left(1+\frac{1}{\beta}\right) f^{i v(k+1)}+\operatorname{Re} f^{(k)} f^{\prime \prime \prime(k+1)}-\left(M+\operatorname{Re} f^{\prime(k)}\right) f^{\prime \prime(k+1)}-\operatorname{Re} f^{\prime \prime(k)} f^{\prime(k+1)}+\operatorname{Re} f^{\prime \prime \prime(k)} f^{(k+1)} \\
& =\operatorname{Re}\left(f^{\prime \prime \prime(k)} f^{(k)}-f^{\prime \prime(k)} f^{\prime(k)}\right) .
\end{aligned}
$$

Now equation (27) provides a system of linear ODEs, where $f^{k}$ denotes the solution of $k^{\text {th }}$ equation. For linear ODEs solution, we replace derivatives with central differences which gives rise to the following linear system: 


$$
B f^{(k+1)}=C, B \equiv B_{n \times n}\left(f^{(k)}\right), C \equiv C_{n \times 1}\left(f^{(k)}\right),
$$

where $n$ is the number of grid points. Also equations (10) and (11) are linear in $\theta$ and $\phi$, and therefore, sequences $\left\{\theta^{(k)}\right\}$ and $\left\{\phi^{(k)}\right\}$ are determined from:

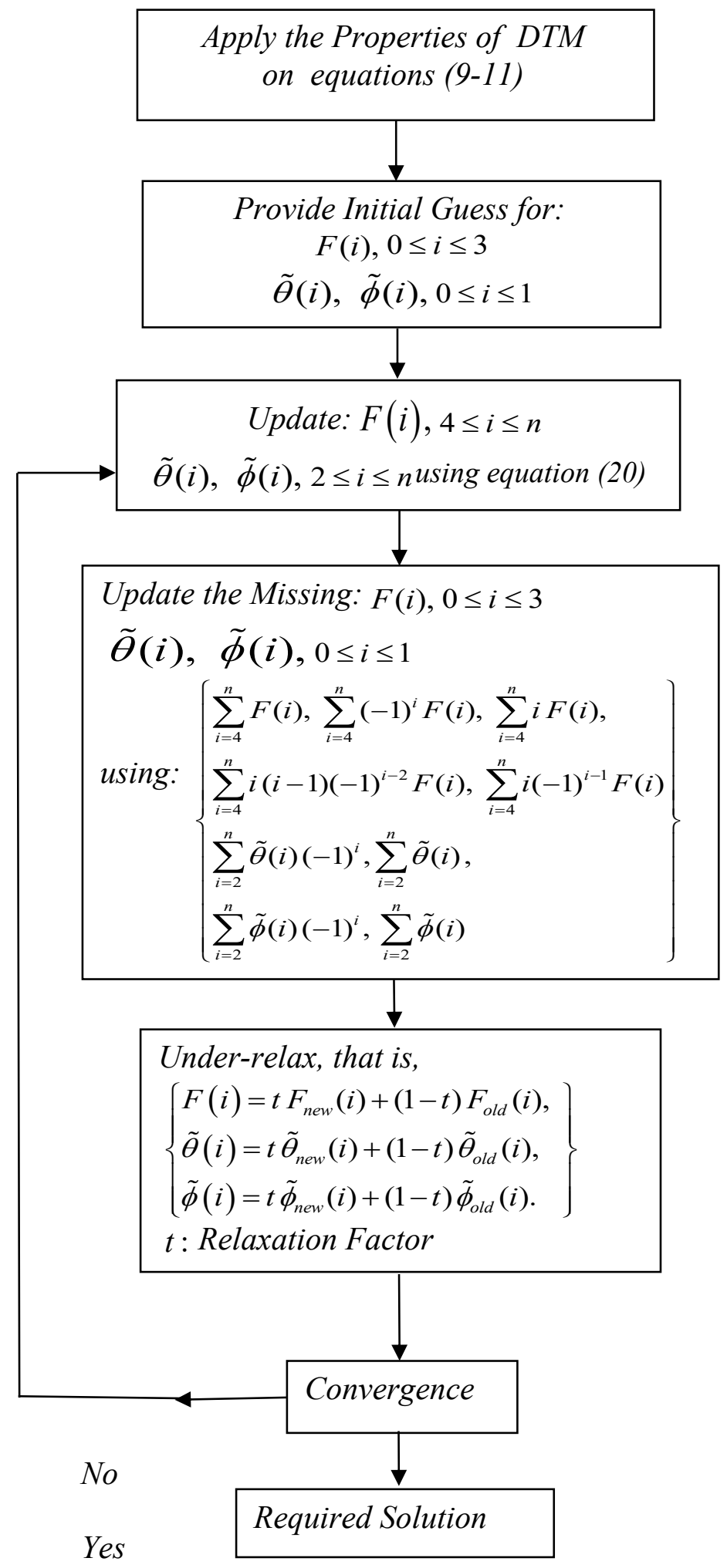

Fig. 2. Phase diagram for the DTM. 


$$
\begin{aligned}
& \theta^{(k+1)^{\prime \prime}}+\operatorname{Pr} R f^{(k+1)} \theta^{(k+1)^{\prime}}=0, \\
& \phi^{(k+1)^{\prime \prime}}(\eta)-\operatorname{Re} S c\left(\begin{array}{l}
\operatorname{Cr} \phi^{(k+1)}(\eta) \\
-f^{(k+1)}(\eta) \phi^{(k+1)^{\prime}}(\eta)
\end{array}\right)=0,
\end{aligned}
$$

where $f^{(k+1)}$ is considered to be known in the above equations and its derivatives are approximated by the central differences. The system of linear equations arising from equations (29-30), after using the central differences are thus solved iteratively by the SOR method.

\section{Results and Discussion}

The behaviour of temperature, velocity and concentration fields across the channel for different values of the parameters (such as $M, \operatorname{Re}, \beta, \lambda, \operatorname{Pr}$ and $\mathrm{Sc}$ ) is discussed, and the results are illustrated in terms of graphs. To illustrate the accuracy of present schemes, comparison of the results for the normal velocity $f(\eta)$, streamwise velocity $f^{\prime}(\eta)$ and concentration $\phi(\eta)$ is presented in Figure 3. It is obvious that the results are found in excellent agreement.

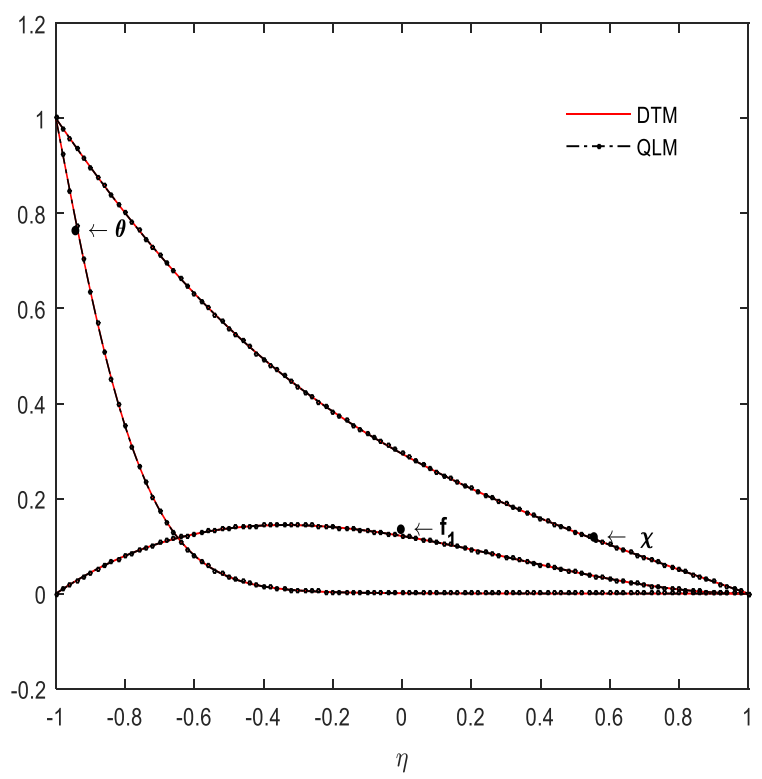

Fig. 3. Comparison of DTM solution with QLM

Figures 4-7, show variation of $f(\eta), f^{\prime}(\eta), \theta(\eta)$ and $\phi(\eta)$ for various values of magnetic parameter $M$. Apparently present phenomena happen when magnetic field creates the opposing or resistive force to the flow direction, which is called Lorentz force. This resistive force slows down motion of the fluid and raises the fluid temperature. Temperature of Casson fluid increases significantly as compared with the Newtonian fluid. Streamwise velocity decreases near the lower wall of the channel whereas an opposite behavior is noticed near the upper wall. A direct consequence of the imposed magnetic field is the increase in temperature and concentration. 


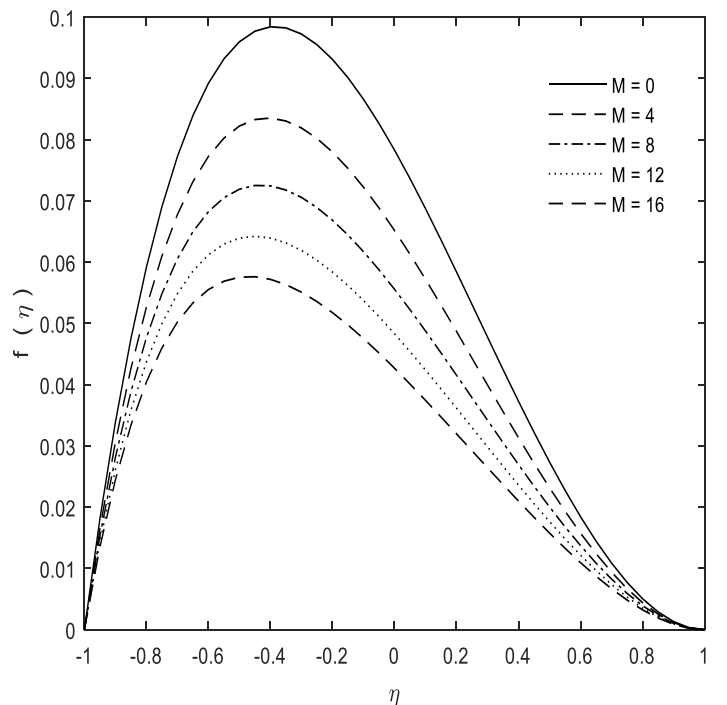

Fig. 4. Normal velocity for $\beta=1, \lambda=0.6, \operatorname{Re}=20, \operatorname{Pr}=2, S c=3, C r=0.01$ and various $M$.



Fig. 5. Streamwise velocity for $\beta=1, \lambda=0.6, \operatorname{Re}=10, \operatorname{Pr}=2, S c=0.8, C r=0.01$ and various $M$.

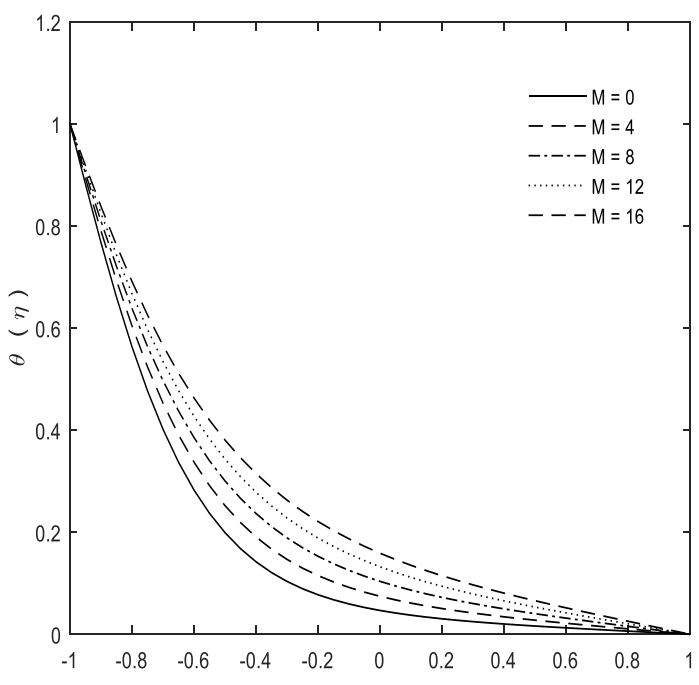

Fig. 6. Temperature for $\beta=1, \lambda=0.6, \operatorname{Re}=10, \operatorname{Pr}=2, S c=0.8, C r=0.01$ and various $M$. 


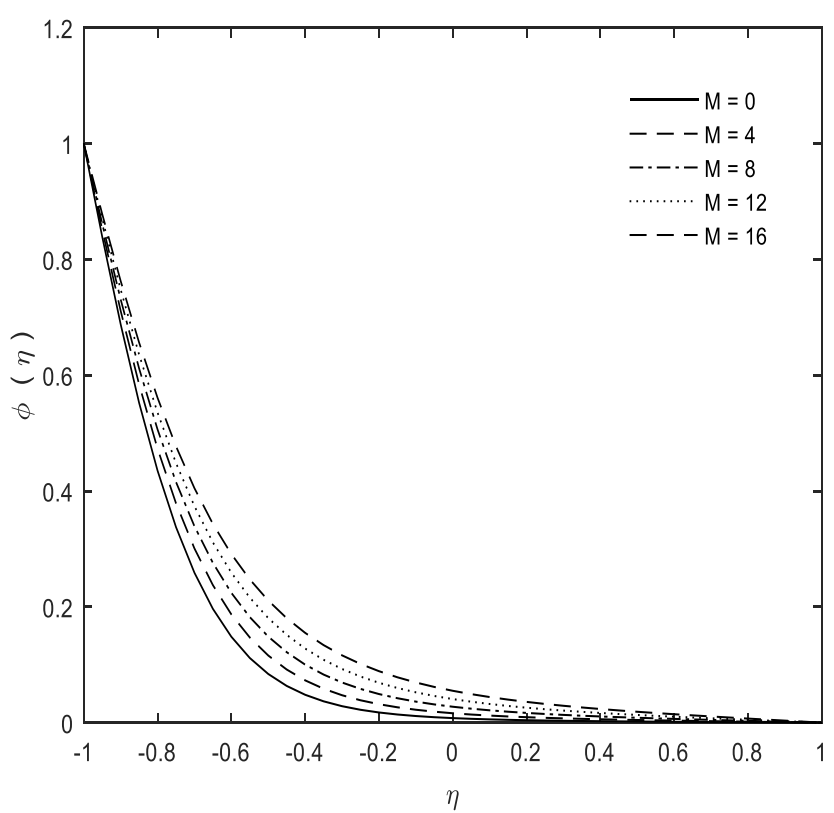

Fig. 7. Concentration for $\beta=1, \lambda=0.6, \operatorname{Re}=10, \operatorname{Pr}=2, S c=0.8, C r=0.01$ and various $M$.

The influence of the Reynolds number $R e$ on the velocity, concentration and temperature profiles is shown in Figures 8-11. Increasing Re decreases the normal velocity distribution across the channel. On the other hand, the streamwise velocity profiles are influenced by $R e$ in opposite way near walls of channel. The concentration and temperature distributions decrease across whole domain. The Reynolds number also tends to mitigate the flattened character of concentration profiles.

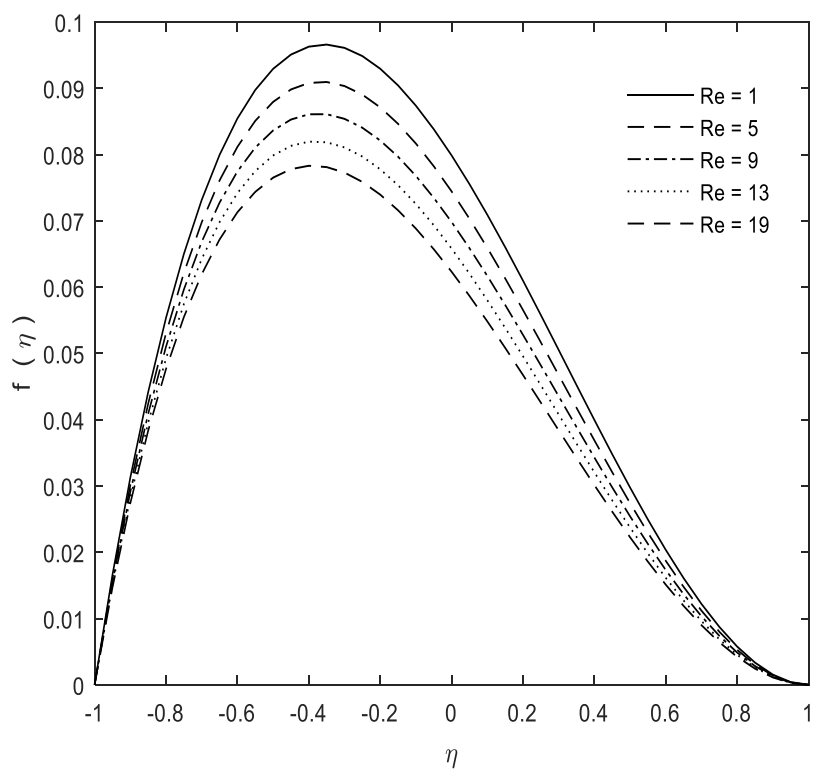

Fig. 8. Normal velocity for $\beta=1.2, \lambda=0.8, M=2, \operatorname{Pr}=4, S c=3.5, C r=0.01$ and various $\operatorname{Re}$. 




Fig. 9. Streamwise velocity for $\beta=1.2, \lambda=0.8, M=2, \operatorname{Pr}=4, S c=3.5, C r=0.01$ and various Re.

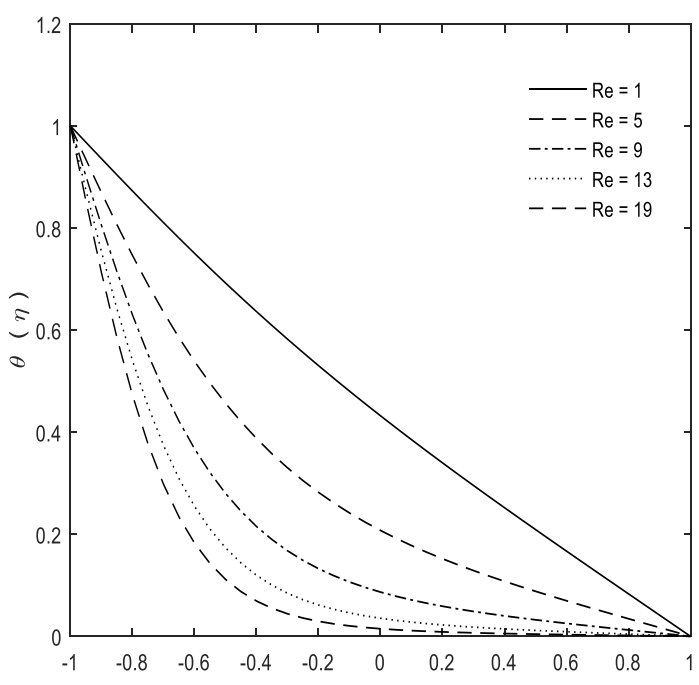

Fig. 10. Temperature for $\beta=1.2, \lambda=0.8, M=2, \operatorname{Pr}=4, S c=3.5, C r=0.01$ and various $\operatorname{Re}$.

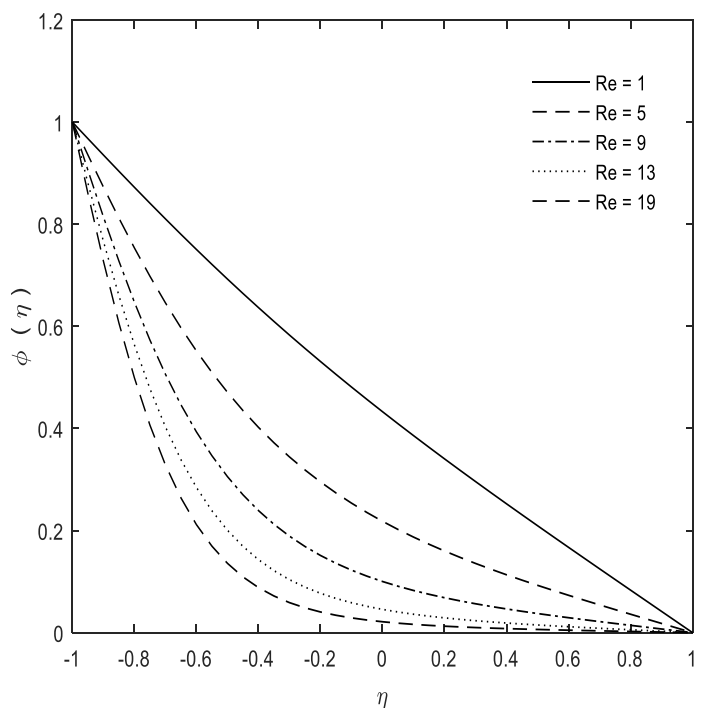

Fig. 11. Concentration for $\beta=1.2, \lambda=0.8, M=2, \operatorname{Pr}=4, S c=3.5, C r=0.01$ and various Re. 
Figures 12-15 depict the influence of the slip parameter $\lambda$ on the various profiles. The presence of slip parameter decreases normal velocity. Moreover, increase in the strength of the slip factor results in reduction of streamwise velocity near the lower wall while streamwise velocity is raised near the upper wall of the channel. The concentration and temperature profiles are also lowered.

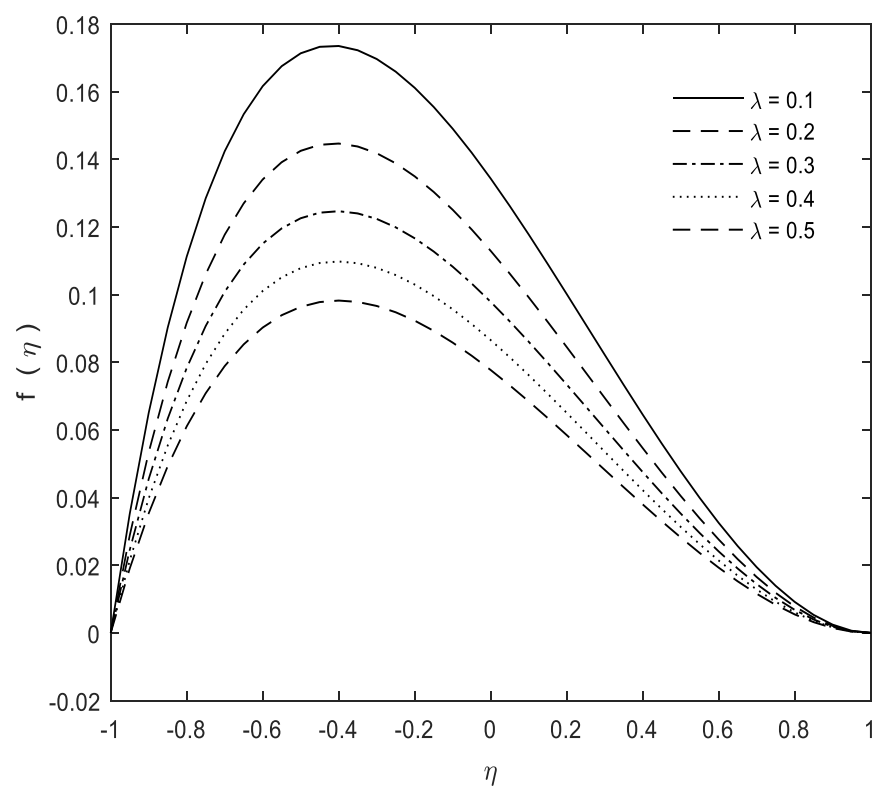

Fig. 12. Normal velocity for $\beta=1, M=5, \operatorname{Re}=10, \operatorname{Pr}=2, S c=0.8, C r=0.01$ and various $\lambda$.

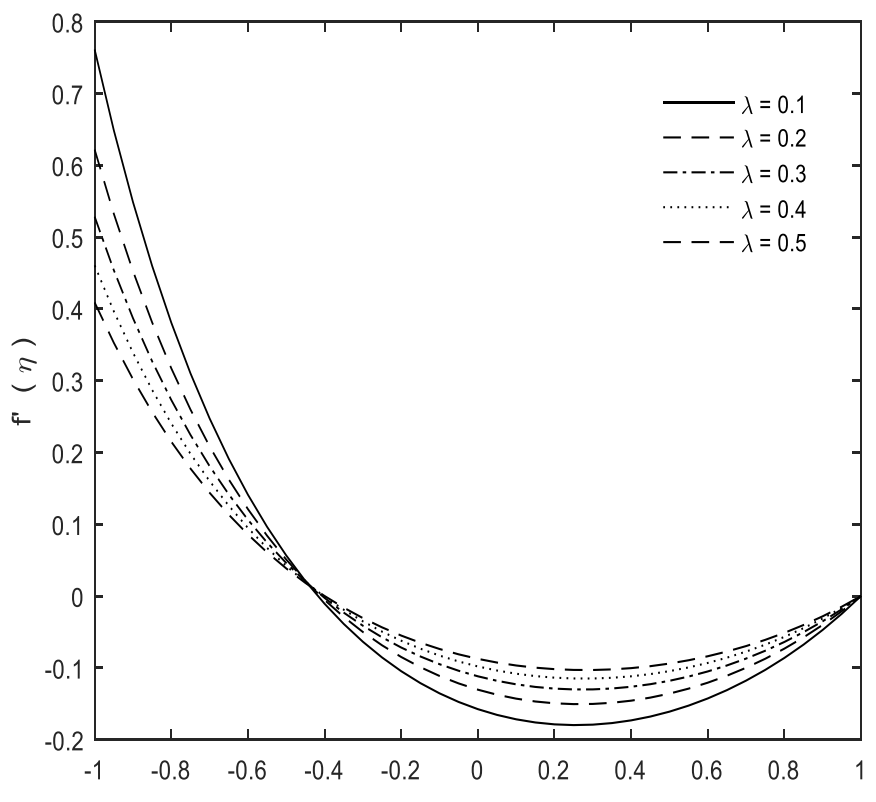

Fig. 13. Streamwise velocity for $\beta=1, M=5, \operatorname{Re}=10, \operatorname{Pr}=2, S c=0.8, C r=0.01$ and various $\lambda$. 


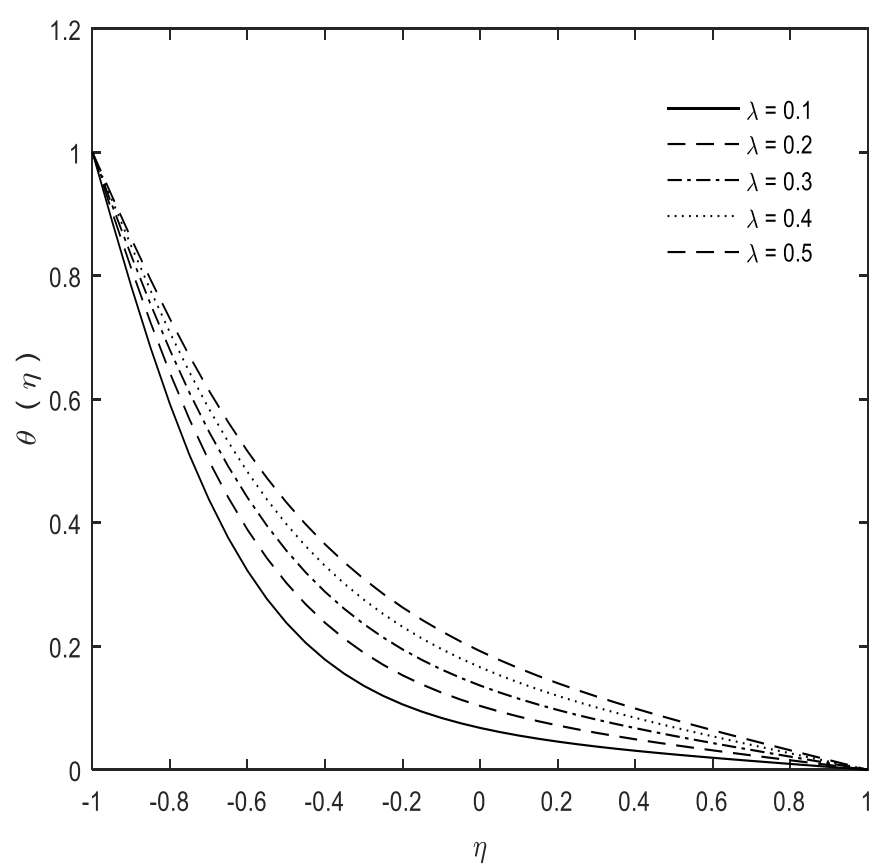

Fig. 14. Temperature for $\beta=1, M=5, \operatorname{Re}=10, \operatorname{Pr}=2, S c=0.8, C r=0.01$ and various $\lambda$.

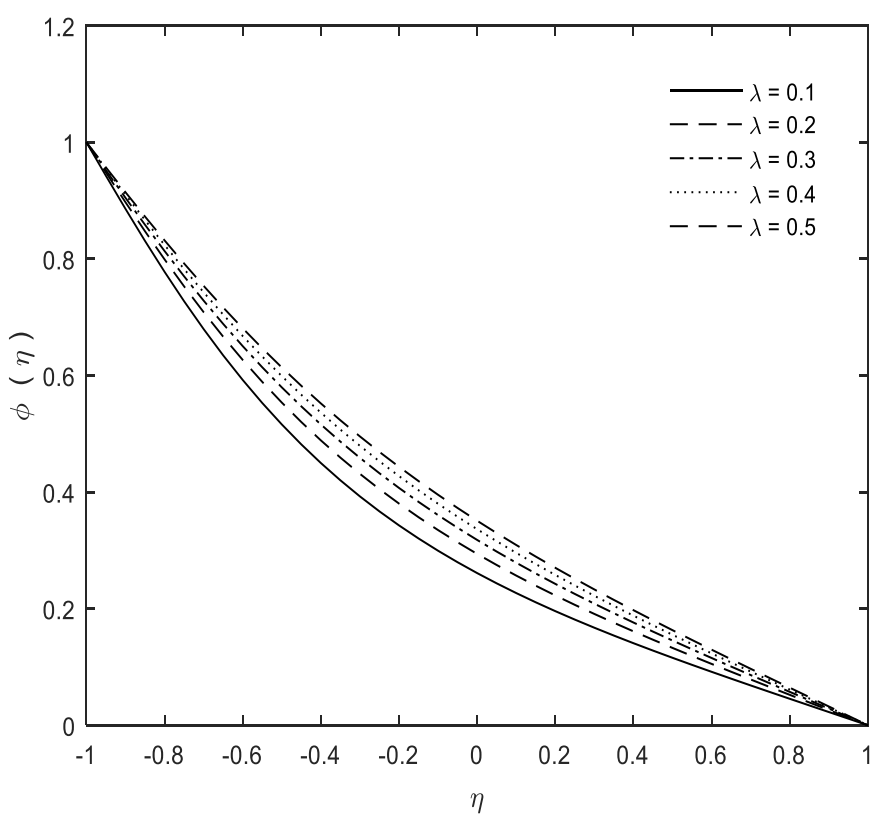

Fig. 15. Concentration for $\beta=1, M=5, \operatorname{Re}=10, \operatorname{Pr}=2, S c=0.8, C r=0.01$ and various $\lambda$.

The impact of the variation in the Casson parameter $\beta$ on the flow, concentration and temperature fields is shown in Figures 16-19. The influence of increasing values of $\beta$ is to reduce the normal velocity. The fluid streamwise velocity is also decreasing at the lower wall of channel with increasing Casson parameter. The fluid acts as Newtonian fluid as $\beta$ becomes significantly large. Figures 18-19 show that the heat and concentration profiles rise with the increasing values of the Casson parameter $\beta$. 


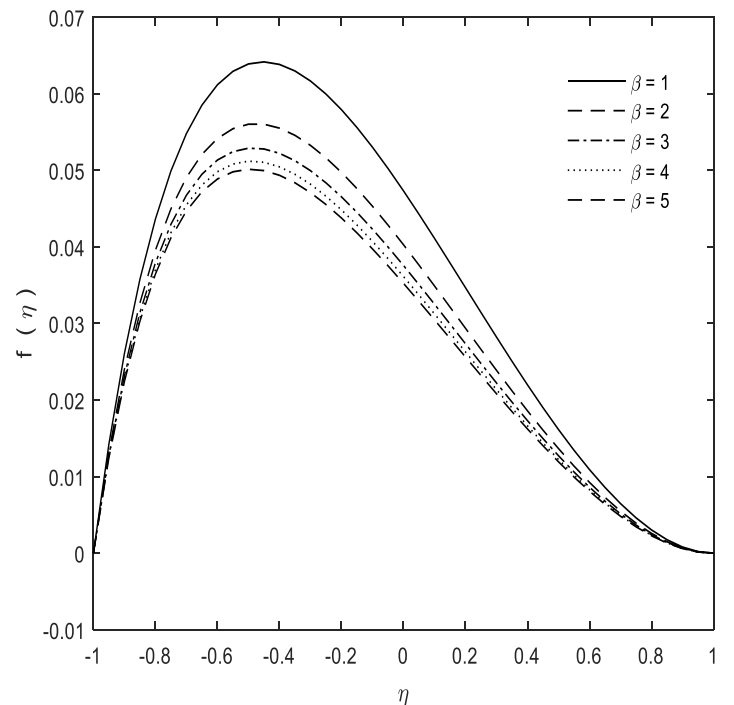

Fig. 16. Normal velocity for $\lambda=0.6, M=5, \operatorname{Re}=50, \operatorname{Pr}=1, S c=0.8, C r=0.01$ and various $\beta$.

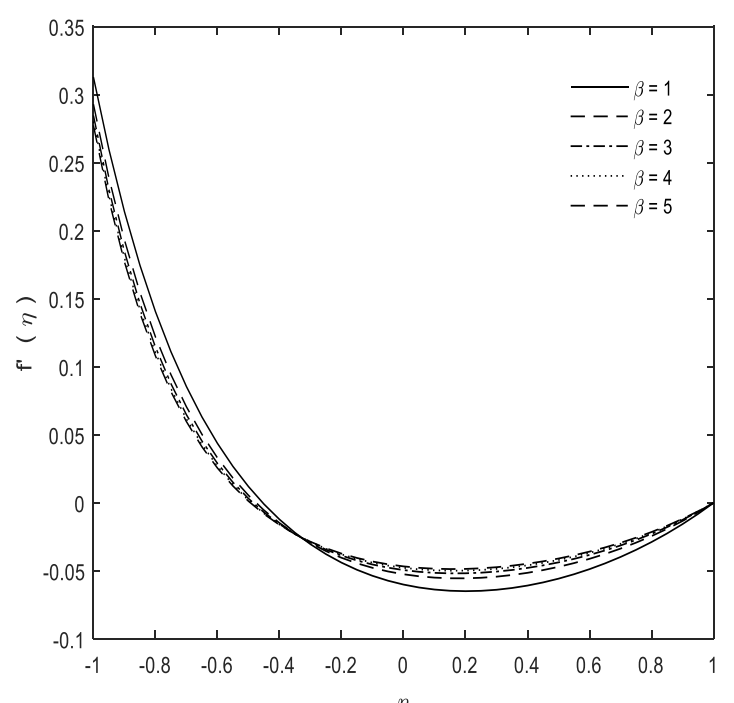

Fig. 17. Streamwise velocity for $\lambda=0.6, M=5, \operatorname{Re}=50, \operatorname{Pr}=1, S c=0.8, C r=0.01$ and various $\beta$.

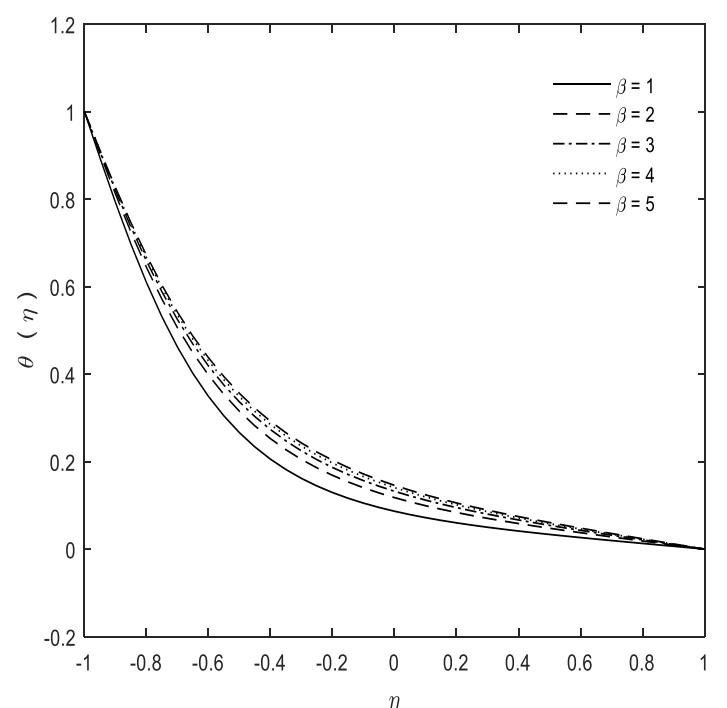

Fig. 18. Temperature for $\lambda=0.6, M=5, \operatorname{Re}=50, \operatorname{Pr}=1, S c=0.8, C r=0.01$ and various $\beta$. 


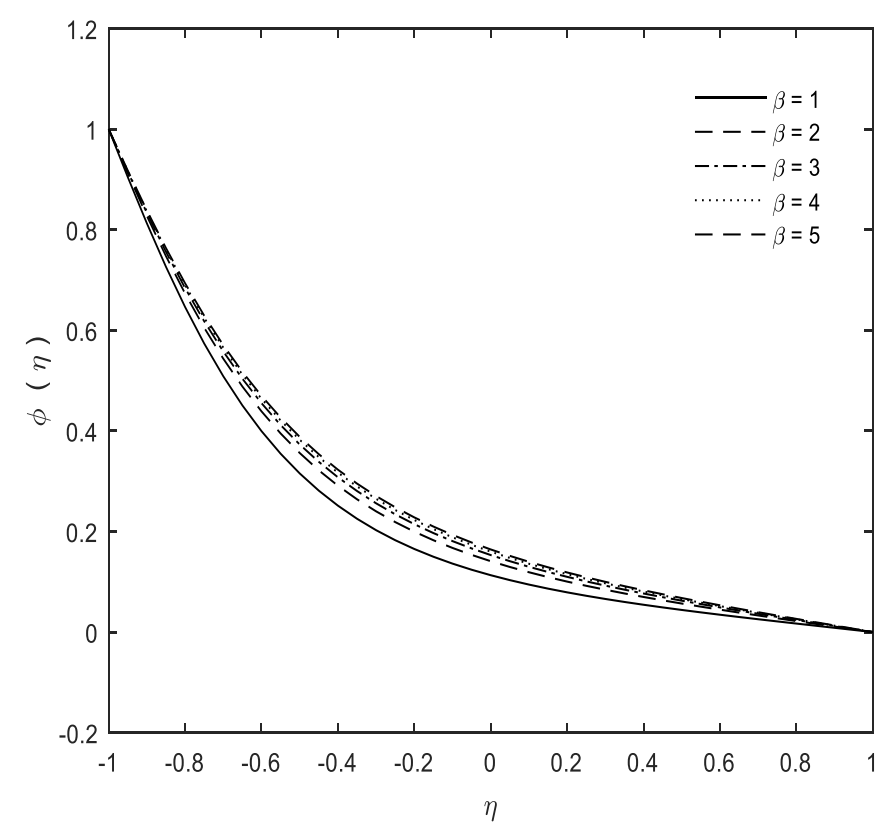

Fig. 19. Concentration for $\lambda=0.6, M=5, \operatorname{Re}=50, \operatorname{Pr}=1, S c=0.8, C r=0.01$ and various $\beta$.

Figure 20 represents how thermal distribution across the channel is affected by the Prandtl number Pr. Increase in the magnitude of Pr, decreases the fluid temperature. Fluids at lower Pr have high thermal conductivities (and thicker thermal boundary layers). Therefore, heat can diffuse from the channel walls more rapidly. As a result, Pr can be used to increase rate of cooling.

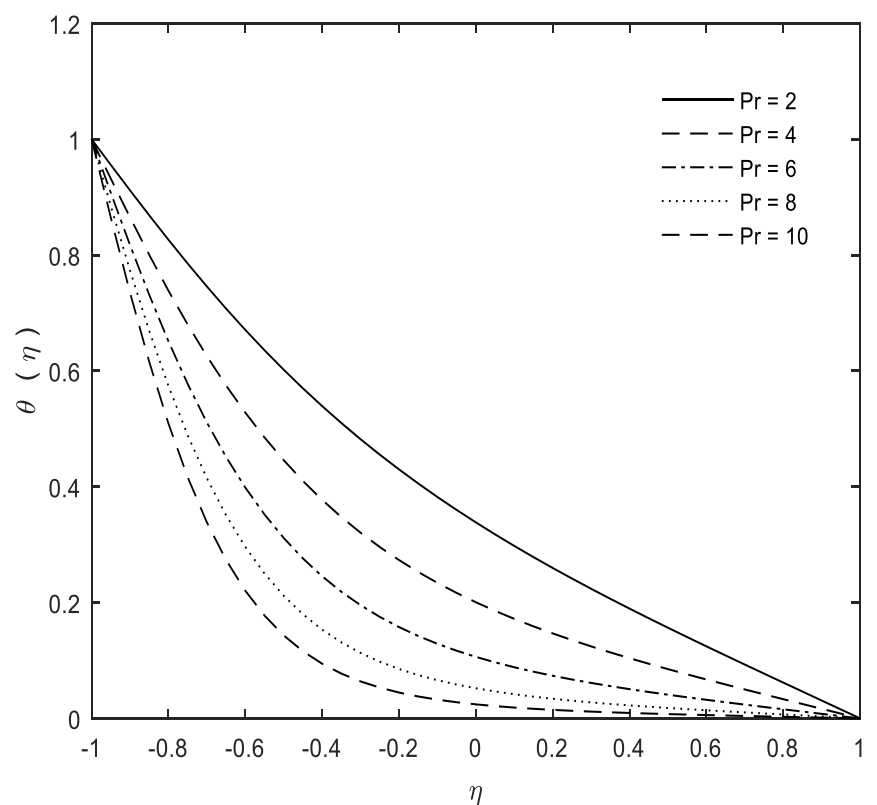

Fig. 20. Temperature for $\lambda=0.6, M=5, \operatorname{Re}=5, \beta=1, S c=0.8, C r=0.01$ and various $\operatorname{Pr}$.

It is evident from Figures 21-22 that increase in $\mathrm{Cr}$ and $\mathrm{Sc}$ significantly lowers the concentration profiles. 


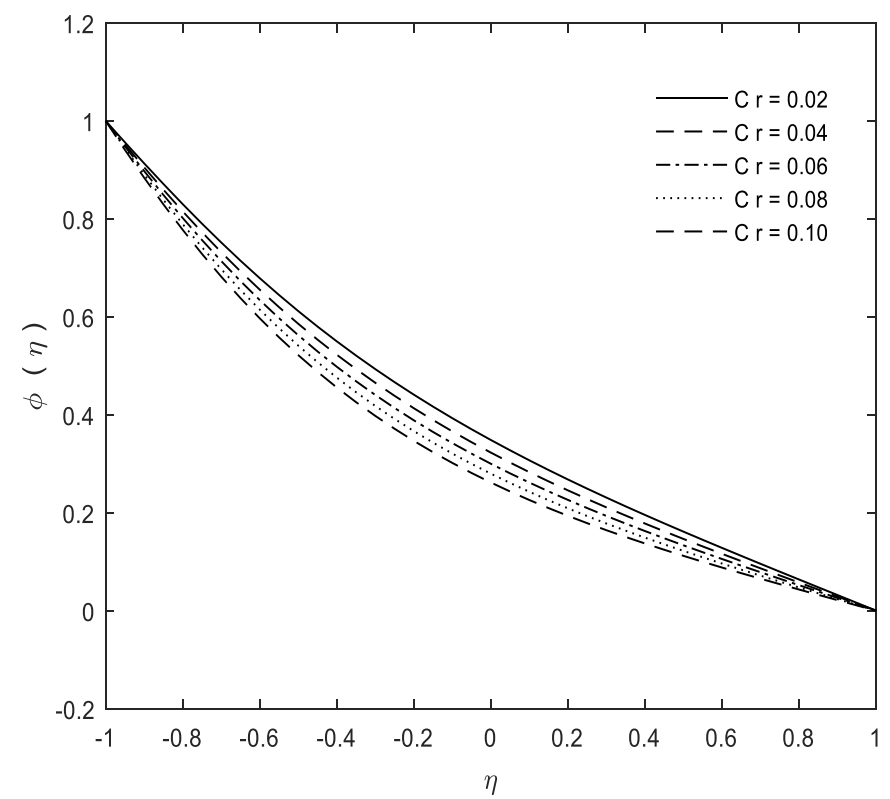

Fig. 21. Concentration for $\lambda=0.6, M=5, \operatorname{Re}=10, \beta=1, S c=0.8, \operatorname{Pr}=2$ and various $C r$.

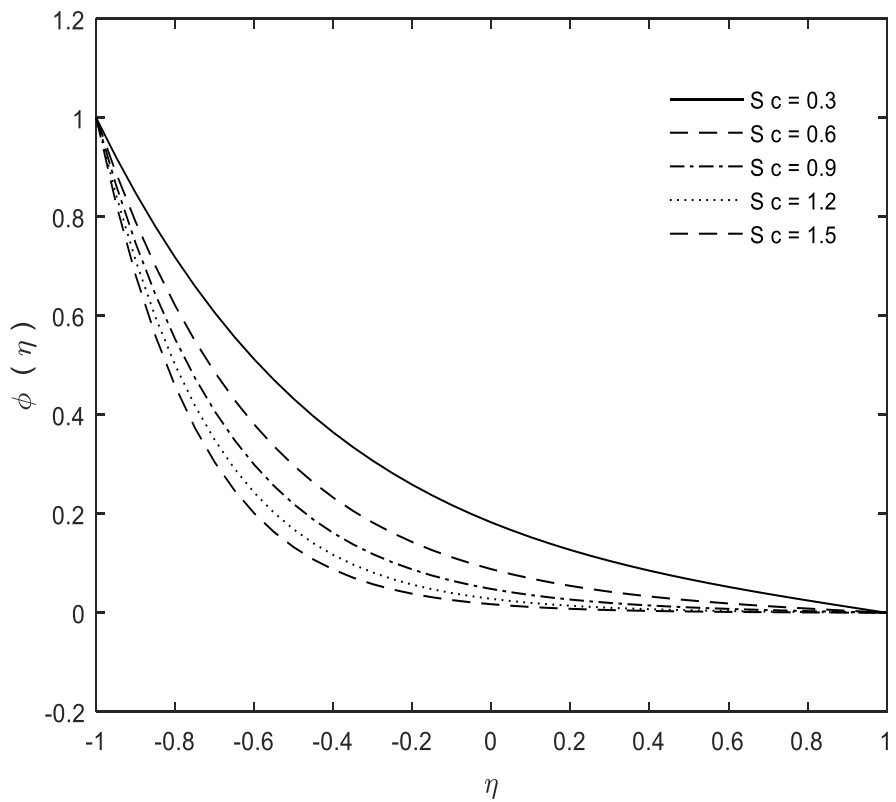

Fig. 22. Concentration for $\lambda=0.6, M=5, \operatorname{Re}=10, \beta=1, C r=0.8, \operatorname{Pr}=2$ and various $S c$.

\section{Conclusions}

In the present study, differential transform technique (DTM) has been successfully utilized for investigating the problem of Casson fluid flow in a channel with one wall at rest and the other shrinking with a slip velocity. It has been noted that magnetic parameter, Casson parameter and slip parameter increase the temperature and concentration distributions while slowing down the flow. The Prandtl number has been noted to increases the cooling rate of the flow. 


\section{References}

Abazari, R. \& Borhanifar, A. (2010). Numerical study of the solution of the Burgers and coupled Burgers equations by a differential transformation method. Computers and Mathematics with Applications, 59: 2711-2722.

Ahmed, F.AL-F. (2006). Numerical study of conjugate heat transfer in enclosures with fins attached to a vertical side wall. Kuwait Journal of Science, 33 (2):205-218.

Ali, K. Ashraf, M. \& Jameel, N. (2014). Numerical simulation of magnetohydrodynamic micropolar fluid flow and heat transfer in a channel with shrinking walls. Canadian Journal of Physics, 92:987-996.

Ali, K. \& Ashraf, M. (2014). Numerical simulation of the micropolar fluid flow and heat transfer in a channel with a shrinking and a stationary wall. Journal of Theoretical and Applied Mechanics, 52(2):557-569, Warsaw.

Ali, K. Ahmad, S. \& Ashraf, M. (2015). Numerical simulation of MHD pulsatile flow of a bio fluid in a channel. AIP Advances, doi: 10.1063/1.4928574.

Aman, F., Ishak, A. \& Pop, I. (2011). Mixed convection boundary layer flow near stagnation point on vertical surface with slip. Applied Mathematics and Mechanics, 32: 15991606.

Ashraf, M. Kamal, M.A. \& Syed, K.S. (2009). Numerical study of asymmetric laminar flow of micropolar fluids in a porous channel. Computers and Fluids,38:1895-1902.

Ashhab, S.A. (2019). Asymptotic behavior and existence of similarity solutions for a boundary layer flow problem. Kuwait Journal of Science,46 (2):13-20.

Ayaz, F. (2004). Solutions of the systems of differential equations by differential transform method. Applied Mathematics and Computation, 147:547-567.

Batra, L. \& Kandasamy, A. (2006). Entrance region flow of a Casson fluid in a straight channel. Polymer-Plastics Technology and Engineering, 31:527-540.

Bhattacharyya, K. Mukhopadhyay, S. \& Layek, G.C. (2011). Slip effects on boundary layer stagnation point flow and heat transfer towards a shrinking sheet. International Journal of Heat and Mass Transfer, 54:308-313.

Chen, C.K. \& Ho, S.H. (1999). Solving partial differential equations by twodimensional differential transform method. Applied Mathematics and Computation, 106:171-170. 
Eldabe, N.T.M., Agoor, B.M. \& Alame, H. (2014). Peristaltic motion of non-Newtonian fluid with heat and mass transfer through a porous medium in channel under uniform magnetic field. Hindawi Publishing Corporation Journal of Fluids doi:org/10.1155/2014/525769.

Fang, T., Zhang, J. \& Yao, S. (2009). Slip MHD viscous flow over a stretching sheet an exact solution. Communications in Nonlinear Science and Numerical Simulation, 14:3731-3737.

Fang, T., Yao, S., Zhang, J. \& Aziz, A. (2010). Viscous flow over a shrinking sheet with a second order slip flow model. Communications in Nonlinear Science and Numerical Simulation, 15:1831-1842.

Fereidoon, A. Davou dabadi, M.R. Yaghoobi, H. \& Ganji, D.D. (2010). Application of homotopy perturbation method and differential transformation method to determine displacement of damped system with nonlinear spring. World Applied Science Journal, 9: 681-688.

Hassan, I.H.A.H. (2002). On solving some eigen value problems by using a differential transformation. Applied Mathematics and Computation, 127(1):1-22.

Haritha, A. \&Sarojamm, G. (2014). Effect of thermal radiation on heat and mass transfer in MHD flow of a Casson fluid over a stretching surface with variable thermal Conductivity. International Journal of Applied Mathematics, 29:1287 -1292.

Hafiz A. W., Sajjad H., Saira B. \& Muhammad N. (2016). Mixed convection flow of PowellEyring fluid over a stretching cylinder with Newtonian heating. Kuwait Journal of Science, 43(3):1-13.

Joneidi, A.A., Ganji, D.D. \& Babaelahi, M. (2009). Differential transformation method to determine fin efficiency of convective straight fins with temperature dependent thermal conductivity. International Communications in Heat and Mass Transfer, 36(7):757-762.

Majeed, A.AL-J. \& AL-Zahraa, J.A.N. (2020). Three iterative methods for solving Jeffery-Hamel flow problem. Kuwait Journal of Science, 47 (1):1-13.

Mridula G. \& Pratibha M. (2013). Analytical solution of the reaction diffusion equation with space time fractional derivatives by means of the generalized differential transform method. Kuwait Journal of Science, 40 (1):23-34.

Momania, S. \& Suat Ert"urk, V. (2008). Solutions of nonlinear oscillators by the modified differential transform method. Computers and Mathematics with Applications, 55: 833-842.

Mukhopadhyay, S. \& Andersson, H.I. (2009). Effects of slip and heat transfer analysis of flow over an unsteady stretching surface. Heat and Mass Transfer, 45:1447-1452. 
Ramesh, K. \& Devaker, M. (2015). Some analytical solutions for the flows o Casson fluid with slip boundary conditions. Ain Shams Engineering Journal, 6:967-975.

Raju, C.S.K., Sandeep, N., Sugunamma, V., Jayachandra Babu, M. \& Ramana Reddy, J.V. (2016). Heat and mass transfer in magnetohydrodynamic Casson fluid over an exponentially permeable stretching surface. Engineering Science and Technology, an International Journal, 19(1):45-52.

Sarojamma, G., Vasundhara, B. \& Vendabai, K. (2014). MHD Casson fluid flow, heat and mass transfer in a vertical channel with stretching walls. International Journal of Scientific and Innovative Mathematical Research (IJSIMR), 2(10):800-810.

Sheikholeslami, M. Ashorynejad, H.R. Ganji1, D.D. \& Rashidi, M.M. (2014). Heat and mass transfer of a micropolar fluid in a porous channel. communications in Numerical Analysis (ISPCS), doi:10.5899/2014/cna-00166.

SI, X.H., Zheng, L.C. Zhang, X.X. \& Chao, Y. (2010). Analytic solution to the micropolar fluid flow through a semi porous channel with an expanding or contracting wall. Applied Mathematics and Mechanics (English Edition), 31:1073-1080.

Wang, C. Y. (2009). Analysis of viscous flow due to a stretching sheet with surface slip and suction. Nonlinear Analysis Real World Applications, 10:375-380.

Wahab, H.A., Hussain, S., Bhatti, S. \& Naeem, M. (2016). Mixed convection flow of PowellEyring fluid over a stretching cylinder with Newtonian heating. Kuwait Journal of Science, 43(3):1-13.

Zhou, J.K. (1986). Differential transformation and its applications for electrical circuits. Hauzhong university press, wuhan china, (in chinese).

$\begin{array}{lr}\text { Submitted: } & 13 / 11 / 2019 \\ \text { Revised: } & 04 / 03 / 2021 \\ \text { Accepted: } & 06 / 03 / 2021 \\ \text { DOI: } 10.48129 / \text { kjs.v49i1.8613 }\end{array}$

